\title{
A bibliometric analysis of scientific literature on cloud manufacturing
}

\author{
Huixing Nie ${ }^{a}$, Aihui Sun ${ }^{\text {b,* }}$ Jinhong Zhong \\ Hefei University of Technology, Hefei, Anhui, China \\ a674863232@qq.com, bappers@163.com, cjinhong.zhong@163.com
}

Keywords: Cloud manufacturing; Knowledge mapping; CiteSpace; Bibliometric analysis

Abstract: Cloud manufacturing is the result of cross-convergence of advanced information technology, manufacturing technology, and emerging Internet of Things technologies, which is the embodiment of the concept of manufacturing service. In recent years, the research on cloud manufacturing has been growing rapidly. It is a significant work to analyze the core authors, core institutions, field hot spots and development directions from a quantitative point of view, which might be provide quantitative references for follow-up research. We selected 463 scientific literature which documented from 2010 to 2017 on the Web of Science and conducted a bibliometric analysis of this papers. Using the visual network analysis tool CiteSpaceIII, this paper draws a scientific knowledge map of cloud manufacturing research and reveal the research status of cloud manufacturing in an intuitive way, including the annual output of papers, the number of authors in a year, the annual input-output ratio of papers and other time distribution, institutions, author and other spatial distribution, keyword co-occurrence network, research frontier and other content analysis maps. The results show that the research on cloud manufacturing has a roughly increasing trend, and the number of scientific research achievements and input personnel are increasing either. Scholars such as Zhang L, Tao F and Ren L and institutions such as Beihang Univ, Univ Auckland, Wuhan Univ Technol have published a relatively large number of documents. The number of articles published by Li bo-hu, Tao F, Zhang L, Ren L was cited more frequently and has a high impact. Research hot spots are changing over time, it is diversified with the progress of the times.

\section{Introduction}

With the rapid development of informatization, the continuous integration of manufacturing technology and information technology has resulted in a number of advanced manufacturing models: agile manufacturing, application service provider, manufacturing grid and so on. Under these manufacturing modes, the enterprises have obtained massive amounts of data. Based on this, the researchers proposed the concept of cloud manufacturing by combining new technologies such as cloud computing, Internet of things and virtualization etc[1]. The Li Bohu academician of Beihang University systematically explained the cloud manufacturing in an article titled by cloud manufacturing--the newly service-oriented network-based manufacturing mode. He defines cloud manufacturing like this: cloud manufacturing is a newly service-oriented network-based 
manufacturing mode which organizes manufacturing resources (manufacturing clouds) according to the based manufacturing mode which organizes manufacturing resources (manufacturing clouds) according to the requests of users through the Internet or (and) cloud manufacturing services platform, provide users with various types of demand-oriented manufacturing services[2].

Cloud manufacturing is the result of cross-convergence of advanced information technology, manufacturing technology, and emerging Internet of Things technologies, and is a manifestation of the idea of manufacturing as a service. Cloud manufacturing is an extension and sublimation of manufacturing informatization, and it is also the landing application of cloud computing in the field of manufacturing [3]. Its proposal provides a brand-new solution for enterprise network-based collaborative manufacturing service needs, and it obtains extensive and in-depth research in academic and applied fields. Xu Xun [4] believes that cloud manufacturing is a model that can acquire manufacturing resources (such as manufacturing tools, manufacturing equipment, and manufacturing capabilities) that can be quickly provided and released from resource sharing pools anytime, anywhere, conveniently, and on demand, also can minimize the amount of management workload and the interaction of service providers. Li Chunquan and others [5] believe that cloud manufacturing is an open, dynamic resource sharing and task collaboration platform that is centered on on-demand services, aggregate decentralized heterogeneous resources into cloud nodes, and virtualize manufacturing resources to facilitate the convenience of information access and control. Tao Fei \& Zhang Lin [6] in Beihang University success to add the value and efficiency of cloud manufacturing system resources service by researching the features of cloud manufacturing, designing cloud service structure, modeling cloud service portfolio and so on. The research of cloud manufacturing is not only theoretical and academic, but also widely applied in practice. The EU's seventh framework started the manufacturing cloud project and provided users with configurable manufacturing capability services under the support of a software-as-a-service application[7].The MFG.com platform provides services, manage-ment, and monitoring for the entire process from the emergence of requirements, quotations, and product shipments [8]; Many large-scale group companies such as the China Aerospace Second Institute and CNR (Beiche Group) are developing and building a group enterprise cloud manufacturing platform that use the manufacturing of key products such as aerospace complex products and rail transportation equipment as their application background, and provide services such as demonstration, design, production, simulation, and management for each stage of the manufacturing life cycle [9,10]. To sum up, whether it is theoretical academic or practical application, the research of cloud manufacturing has received more and more attention, which is worthy of further study.

In recent years, the research on cloud manufacturing has been growing rapidly, and the articles on cloud manufacturing review have appeared frequently. There are "Cloud manufacturing: Strategic vision and state-of-the-art" by Wu Dazhong \& Greer J. Matthew et al. [8] Adamson Goran \& Wang Lihui et al. [11]"Cloud manufacturing - a critical review of recent development and future trends"; Qi Ershi et al.'s [12] "Review of Cloud Manufacturing Theory, Technology and Related Applications", Yi Shuping et al.'s [13], "A Review of Cloud Manufacturing Services Based on Lifecycle. These articles are analyzed from a qualitative point of view, subjectivity is too strong, subject to the influence of experts in the field, is not conducive to the objective analysis of the study. There are fewer literatures on objective and quantitative analysis of cloud manufacturing. Quantitative and qualitative analysis are equally important for research in a field and are indispensable.

In order to accurately grasp the development history and practice path of cloud manufacturing, this study from the perspective of quantitative analysis, uses visualization tools to perform quantitative analysis on the collected scientific literature, and visualizes the research results through scientific knowledge maps. The overall status quo, knowledge structure and future development direction of cloud manufacturing research are explored, for follow-up research provides practical 
and valuable reference, and provides important guidance for future practice.

\section{Data sources and research tools}

\subsection{Data source}

In this study, the core collection (including SCI-EXPANDED, SSCI, CPCI-S and CPCI-SSH) of the Web of Science (WOS) database was selected as the source of the literature. The retrieval time was January 6, 2018, and the literature was collected using the search form "TS = (cloud manufactur *)" where "*" represents each word that has the same root as "manufacture". The scope of the subject is limited to related subjects of cloud manufacturing, with a search span ranges from 2010 to 2017, and a total of 471 articles were retrieved. The papers are then screened using their own analytical search tools to select proceedings papers and articles. Finally, 463 articles were obtained as the literature samples of this study.

\subsection{Research tools}

Mapping Knowledge Domains is a graphic used to show the relationship between the development process and structure of scientific knowledge [14]. The concept originated from a seminar organized by the National Academy of Sciences of the United States in 2003. With the development of information visualization, various tools for mapping scientific knowledge maps have come to the fore [15]. CiteSpace is a commonly used information visualization tool developed by Professor Chen Chaomei in Drexel University, USA [16]. It can calculate the number of articles in a specific field and explore the critical path of the field's evolution and knowledge turning points based on co-citation analysis theory and PFNET through a series of visualized maps [17]. In this study, the software was used to describe the statistics and mines data of cited literature and citing in the field of cloud manufacturing, and it draw up the knowledge base map and analyze its research content, evolution path, and front hot spots. In addition, this study also uses the office software Microsoft Office Excel 2016 and citation analysis software Histcite to assist the analysis.

\section{Spatiotemporal Knowledge Map and Its Analysis}

\subsection{Cloud manufacturing research time distribution map}

An important index for measuring the development in a certain field is to study the changes of the number of papers, and to count the number of documents and to draw the corresponding growth curve plays an important role in evaluating the stage in which the field is located and forecasting its development trend. As shown in Figure 1, the year-on-year changes in the number of documents from 2010 to 2017 show an overall upward trend. The research on cloud manufacturing originated in 2010, and from 2010 to 2014, the research is in an initial stage. In this stage, the number of scientific literature has been growing rapidly, reaching 85 in 2014. While the trend was flat in 2015, the number of published papers are as many texts as in 2014. In 2016, the number of published articles increased to 103, reaching a new peak. The volume of papers issued in 2017 was 93, 10 fewer than in 2016, but 8 more than in 2015. In general cloud manufacturing research showed a linear trend of growth.

It can be seen that the degree of attention of a field is from the input of scientific research personnel this field. The change trend of the input amount of the author in the year is shown in Figure 2 below. The change trend of the annual input amount of the author and the change trend of the number of scientific literature are generally the same, and both show a linear increase. The number of authors in the study was 6 in 2010, and by 2016 it had grown to 383, an increase of more 
than 60 times. The author of the research on cloud manufacturing has more and more input, and it is becoming more and more important.

In order to investigate the input and output ratio of scientific researchers in the cloud manufacturing field, this study count the participants of a single paper and obtain the change trends in the ratio of personnel participating in a single paper. The result is shown in figure 3. From 2010 to 2011, the number of participants in a single paper was changing rapidly, which shows that it was 3.00 in 2010, and become a current peak,4.11 in 2011.From 2012 to 2017, there was a wave of change, but the change was small. In these six years, the average number of participants in each paper was 3.60. The number of co-authors in cloud manufacturing research is high.

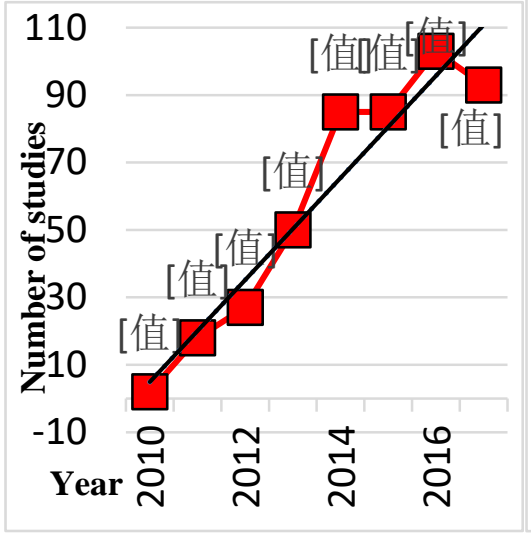

Figure 1. published articles

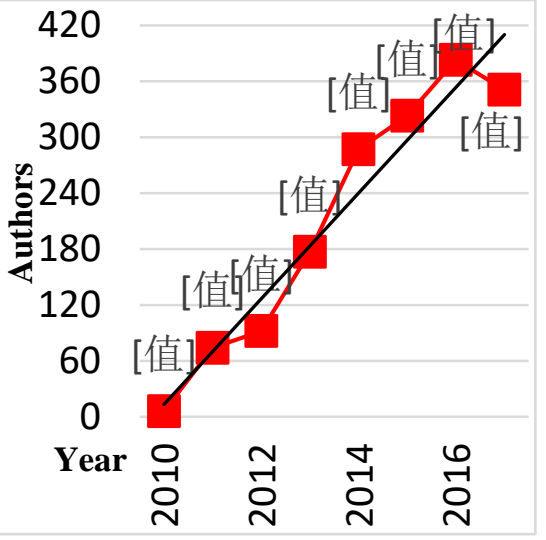

Figure 2. Authors

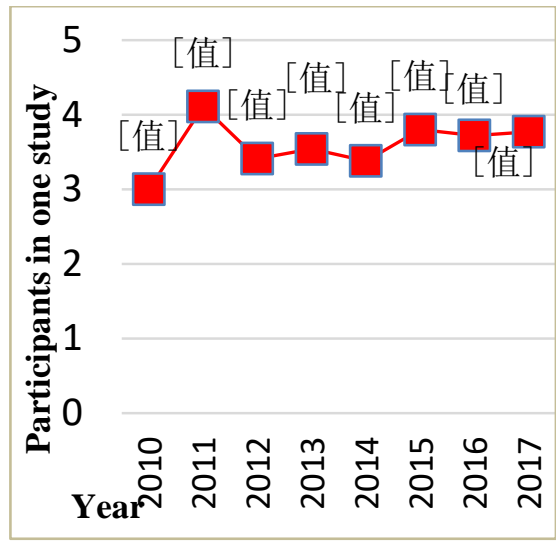

Figure 3. Co-authors per article

\subsection{Cloud Manufacturing Research spatial distribution map}

\subsubsection{Institutional distribution}

In order to find out the core academic groups and institutions that are involved in cloud manufacturing research, this paper lists the number of papers published by various research institutes in cloud manufacturing research, involving a total of 287 units. The top 13 high production institutions are shown in Table 1, in which shows that Beihang Univ occupies the first place in the number of publications with greater advantages, indicating that this agency has a strong research potential and academic communication awareness in the cloud manufacturing research. The second one is Univ Auckland, whose published papers is 32 more than Wuhan Univ Technol. KTH Royal Inst Technol, Minist Educ and Zhejiang Univ ranked 4th, 5th and 6th with 25, 19 and 16 papers respectively. Subsequent seven agencies in the volume of paper differ in the range of 1-3 articles, 
indicating that the research of cloud manufacturing is widely concerned by international academic institutions.

There are two divisions of citation frequency in the Histcite system: LCS and GCS. LCS (Local Citation Score) refers to the citation frequency of an article in the local database, whereas GCS (Global Citation Score) refers to the citation frequency of an article in the Web of Science (WOS) database. TLCS and TGCS are used to represent the total citation frequency in terms of LCS and GCS, respectively [18]. As shown in Table 1, Zhejiang Univ volume of 16, a TLCS of 6, a GLCS of 21, and Tsinghua Univ issued a document with a volume of 9, a TLCS of 51, and a GLCS of 102. In comparison, the research influence of Zhejiang Univ in this field is not as high as Tsinghua University.

Table 1 Issued more than 8 papers (including 8) institutions

\begin{tabular}{|c|c|c|c|}
\hline Institution & No. of published articles & TLCS & TGCS \\
\hline Beihang Univ & 68 & 603 & 1171 \\
\hline Univ Auckland & 32 & 317 & 668 \\
\hline Wuhan Univ Technol & 29 & 61 & 92 \\
\hline KTH Royal Inst Technol & 25 & 60 & 230 \\
\hline Minist Educ & 19 & 64 & 96 \\
\hline Zhejiang Univ & 16 & 6 & 21 \\
\hline Chongaing Univ & 12 & 87 & 118 \\
\hline Beijing Simulat Ctr & 11 & 87 & 186 \\
\hline Beijing Inst Technol & 9 & 25 & 33 \\
\hline Northwestern Polytech & 9 & 17 & 46 \\
\hline Tsinghua Univ & 9 & 51 & 102 \\
\hline Univ Birmingham & 9 & 2 & 7 \\
\hline Univ Hong Kong & 9 & 15 & 47 \\
\hline
\end{tabular}

\subsubsection{Author distribution}

In order to investigate the distribution of authors in cloud manufacturing field, 66 authors who published 4 or more articles were selected from the 886 authors included in the total literature as high yield authors. The top 11 prolific authors and their published volumes are shown in table 2 . In terms of the volume, Zhang L ranks first with 58 papers, and Tao F is followed with 44 articles. Although they differed by 14 in the number of articles published, there was almost no difference between Tao $\mathrm{F}$ (565, 1134) and Zhang L $(566,1136)$ in TLCS and TGCS. Wang LH ranked third with 31 articles, while Ren L and Xu X ranked fourth with 27. Wang LH's TLCS and TGCS are lower than the other two. Subsequent six authors in the volume of paper differ in the range of 1-3 articles, but the paper's TLCS and TGCS have great difference. The top five organizations in the volume of documents in Table 1 basically include the top ten high yield authors, which shows that there are many cloud manufacturing research teams in these institutions and they cooperated in publishing articles. 
Table 2. The number of papers published in more than 10 (including 10) of the author

\begin{tabular}{|c|c|c|c|c|c|c|c|}
\hline Authors & $\begin{array}{c}\text { No. of } \\
\text { published } \\
\text { articles }\end{array}$ & TLCS & TGCS & Authors & $\begin{array}{c}\text { No. of } \\
\text { published } \\
\text { articles }\end{array}$ & TLCS & TGCS \\
\hline Zhang L & 58 & 566 & 1136 & Cheng Y & 15 & 265 & 538 \\
\hline Tao F & 44 & 565 & 1134 & Liu YK & 15 & 91 & 184 \\
\hline Wang LH & 31 & 51 & 185 & Luo YL & 15 & 166 & 290 \\
\hline Ren L & 27 & 210 & 365 & Wang XV & 15 & 82 & 149 \\
\hline Xu X & 27 & 257 & 565 & Xu WJ & 15 & 9 & 15 \\
\hline Liu Q & 18 & 10 & 52 & & & & \\
\hline
\end{tabular}

\section{Knowledge Base Analysis of Cloud Manufacturing Research}

A knowledge base consists of the previous research content and structure of a specific field. It is a means for better understanding the essence of a research front [19]. The research front refers to the development in a research field; therefore, the introduction of a research front constitutes the related knowledge base. Chen [20] redefined the knowledge base (intellectual base) of a research front as the citation trajectory of the research front in the literature.

In order to understand the knowledge base and innovation path of cloud manufacturing research field, using Citespace III conduct the literature co-citation analysis and obtain Literature co-citation network diagram. Co-citation networks are knowledge networks formed in specific situations when two articles are cited by a third article or by several different articles concurrently [21]. Co-citation analysis focuses on the frequency of co-citation by other articles to describe the relationship between the articles; for example, the more likely that two articles are cited in a third article, the closer relationship they have. In other words, the two articles share a similar research background [22]. Through the literature co-citation network, the knowledge base of cloud manufacturing research field can be concretely displayed.

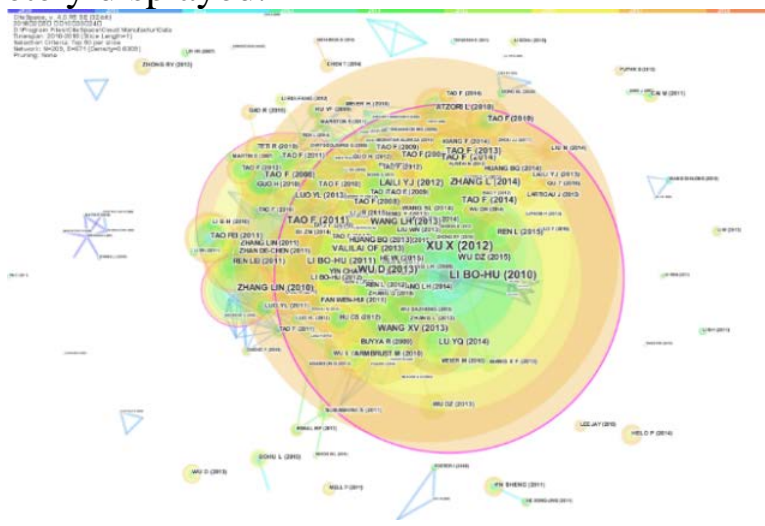

Figure 4. The total cited literature network

In the document co-citation network in Figure 4, each node represents the referenced article, and the node is formed by rings of different colors of rings, the larger the radius, the higher the frequency of citation; The annual color of the rings is warm, indicating that the time it is cited is closer to the present time. There are deep red dots inside the node, which indicates that the reference of this document has changed significantly in some years. The lines between nodes indicate the co-citation relationship, and the thickness indicates the strength of co-citation $[23,24]$.As shown in Figure 4, $\mathrm{Xu} \mathrm{X}$ (2012) published in ROBOTICS AND COMPUTER-INTEGRATED MANUFACTURING a scientific article entitled From Cloud Computing to Cloud Manufacturing 
with the highest citation frequency of 211 and the connection with Tao F (2011) was thicker, and the co-citation relationship was strong, shows that the correlation between the scientific literature published by Xu X (2012) and the scientific literature published by Tao F (2011) is slightly stronger and the topics of the literature are relatively similar. A scientific paper cited by Li Bo-hu (2010) at Computer Integrated Manufacturing Systems entitled Cloud Manufacturing: A New Service-Oriented Networked Manufacturing Model was cited 191 times. This article first puts forward the concept of cloud manufacturing, and gives a detailed explanation, which is the beginning of the field of cloud manufacturing research. The citation frequency of a scientific document published in the PROCEEDINGS OF THE INSTITUTION OF MECHANICAL ENGINEERS PART B-JOURNAL OF ENGINEERING MANUFACTURE by Tao F (2011) was 101, which was similar to that published by Chartrand Gray (2006) and Tao F (2008).

Table 3 Cited documents Cited network nodes related information (center degree> 0.10)

\begin{tabular}{|c|c|c|c|c|c|}
\hline Author & Study name & Year & Journal & Frequency & Centrality \\
\hline $\begin{array}{c}\text { Li-Bo } \\
\mathrm{Hu}\end{array}$ & $\begin{array}{l}\text { Cloud manufacturing:a new } \\
\text { service-oriented networked }\end{array}$ & 2010 & $\begin{array}{l}\text { Computer Integrated } \\
\text { Manufacturing }\end{array}$ & 191 & 0.22 \\
\hline Tао F & $\begin{array}{l}\text { Cloud manufacturing: a computing and } \\
\text { service-oriented manufacturing model }\end{array}$ & 2011 & $\begin{array}{l}\text { P I MECH ENG B-J } \\
\text { ENG }\end{array}$ & 100 & 0.18 \\
\hline Tао F & $\begin{array}{l}\text { Study on manufacturing grid resource } \\
\text { service QoS modeling and evaluation }\end{array}$ & 2009 & $\begin{array}{c}\text { INT J ADV MANUF } \\
\text { TECH }\end{array}$ & 20 & 0.17 \\
\hline $\begin{array}{c}\text { Zhang } \\
\text { L }\end{array}$ & $\begin{array}{l}\text { Key technologies for the construction of } \\
\text { manufacturing cloud }\end{array}$ & 2010 & $\begin{array}{l}\text { Computer Integrated } \\
\text { Manufacturing }\end{array}$ & 41 & 0.15 \\
\hline Ren L & $\begin{array}{c}\text { A methodology towards } \\
\text { virtualisation-based high performance } \\
\text { simulation platform supporting } \\
\text { multidisciplinary design of complex }\end{array}$ & 2011 & $\begin{array}{c}\text { ENTERPRISE } \\
\text { INFORMATION } \\
\text { SYSTEMS }\end{array}$ & 21 & 0.11 \\
\hline
\end{tabular}

It is generally considered that nodes with betweenness centrality greater than 0.10 are more important in the network structure and play a specific role in the evolution of knowledge structure. In figure 4, part of the node color is purple, indicating that the heart is larger. The node related information in the literature co-citation network is given in Table 3, which is ranked Li-BoHu (2010, 0.22), Tao F (2011, 0.18), Tao F (2009, 0.17), Zhang L (2010, 0.15), Ren L (2011, 0.11) according to centrality. These five articles are the core literature of cloud manufacturing research and have a huge impact on the research of cloud manufacturing. Each of the high centrality literature represents the core research area within a certain period of time, and most of the future research will be expanded from these high centrality articles [25]. Through the unremitting efforts of researchers in the field of cloud manufacturing research, the foundation of the knowledge in this field has been formed and developed, which provides a relatively complete knowledge base for the follow-up research of researcher.

\section{Content knowledge map and its analysis}

The research hotspot is the focal point and intensive place of the disciplinary research in a certain period of time, manifested in the emergence of a large number of literature and academic thoughts in a subject issue, and the emergence of a large number of relevant researchers [23]. It is helpful for scholars to grasp the research trends in a subject field and understand the research direction and development situation [26]. However, the key words are the soul manifestation of a scientific literature, which is a highly generalization of the main research contents, main academic thoughts and 
main research methods of the researchers. At the same time, the key words are the research directions and research fields that can reflect the dissertation from the side and are the important indicators of the scientific measurement research [27]. Statistics of keywords and keywords in the scientific literature co-occurrence frequency, the level of frequency can reflect the size of the relevance of the keywords as well as the period of time in a particular area of hot issues. If a certain key word appears repeatedly in the literature of its own field, the research topic represented by this key word is the research focus that is more concerned in this field. The stronger the centrality of a keyword is, the more information flow between the keywords it controls and the more the key words co-occur with other keywords in the literature, so it is likely that the high centrality keywords are a research hotspot in this discipline or field.

In order to investigate the hot spots in the research of cloud manufacturing, the frequency of occurrence of keywords in the literature was calculated, which contained 1473 keywords and 212 kinds of keywords. Among all the key words, high-frequency keywords can best reflect the research hot spots and research trends in specific fields [28]. As shown in Table 4, the first 34 high-frequency keywords with the frequency of words above 10 are selected as the research samples of this paper, and their respective centralities are also listed. In Table 4, the terms such as Cloud Manufacturing, System, Cloud Computing, Model, Design, Management, etc. have a higher word frequency, among them Ontology, Thing, Simulation and other nodes have emergent phenomena. The high centrality keywords are: Cloud Manufacturing (cmfg) (0.27), Cloud Manufacturing (0.27), Cloud Computing (0.22), Management (0.12), Design (0.12) and more. It is generally accepted that nodes with betweenness centricity greater than 0.10 occupy an important position in the network structure, indicating that these keywords are in a prominent position in the co-occurrence network structure and play a crucial role in the expansion of the research direction of cloud manufacturing.

Table 4 The first 34 high-frequency keywords (frequency> 10)

\begin{tabular}{|c|c|c|c|c|c|c|c|}
\hline & Keyword & Freque & Centra & & Keyword & Freque & Centra \\
\hline 1 & Cloud & 293 & 0.27 & 18 & Thing & 19 & 0.01 \\
\hline 2 & System & 82 & 0.09 & 19 & Service & 19 & 0.02 \\
\hline 3 & Cloud Computing & 54 & 0.22 & 20 & Integration & 18 & 0.02 \\
\hline 4 & Model & 40 & 0.11 & 21 & Selection & 18 & 0.03 \\
\hline 5 & Design & 37 & 0.12 & 22 & Internet of Thing & 17 & 0.02 \\
\hline 6 & Management & 31 & 0.12 & 23 & Big Data & 16 & 0.02 \\
\hline 7 & Resource & 31 & 0.07 & 24 & Architecture & 14 & 0.04 \\
\hline 8 & Of the Art & 30 & 0.03 & 25 & Virtualization & 14 & 0.09 \\
\hline 9 & Internet & 29 & 0.03 & 26 & Cloud & 13 & 0.02 \\
\hline 10 & Optimization & 28 & 0.05 & 27 & Grid system & 13 & 0.09 \\
\hline 11 & $\begin{array}{c}\text { Cloud } \\
\text { Manufacturing(cmfg }\end{array}$ & 27 & 0.27 & 28 & Simulation & 12 & 0.00 \\
\hline 12 & Ontology & 26 & 0.00 & 29 & Technology & 12 & 0.04 \\
\hline 13 & Platform & 26 & 0.12 & 30 & Enterprise & 12 & 0.07 \\
\hline 14 & Algorithm & 24 & 0.02 & 31 & Challenge & 11 & 0.05 \\
\hline 15 & Service & 23 & 0.06 & 32 & Virtual Enterprise & 10 & 0.02 \\
\hline 16 & Framework & 21 & 0.02 & 33 & $\begin{array}{l}\text { Service oriented } \\
\text { architecture }\end{array}$ & 10 & 0.01 \\
\hline 17 & Genetic Algorithm & 19 & 0.02 & 34 & Performance & 10 & 0.01 \\
\hline
\end{tabular}

\subsection{Keywords co-occurrence network map}

The above from the perspective of high frequency keywords and centrality, the paper reveals the 
research hotspots of cloud manufacturing, and the following will explain the hot issues of cloud manufacturing from the perspective of keywords co-occurrence map. By Citespace software to analyze the keywords in the literature, the formation of keyword co-occurrence network atlas, as shown in Figure 5. Figure 5 contains 207 nodes, 674 edges, the overall network density of 0.0316 . A node represents a keyword, and the shape of the node is presented as a tree ring. A circle represents a year, the thicker the ring represents the higher the frequency of the keywords in the corresponding year, the larger the node is the greater the frequency of the cumulative occurrence of the keyword. The connection between two nodes indicates that the two keywords appear together. Compared with author's and institution's knowledge map, there are a large number of keywords and more complicated edges. By dragging the nodes in the graph and adjusting the parameters of the control panel, the keyword nodes whose frequency is greater than 8 are displayed with labels. The key words scattered, and no overlap, can clearly show the cloud manufacturing research hot spots. According to Table 4 and Figure 5, analyzing the research hot spots from different perspectives gives similar results.

\subsection{Research frontier keywords sequence diagram}

In order to reflect the use of different keywords at different times, on the basis of Figure 5, we draw the Time Zone view of the key words of cloud manufacturing research on time sheet, as shown in figure 6. In 2010, the concept of cloud manufacturing just is proposed, the number of nodes is very few, indicating fewer scholars who pay close attention to this field in this year, this is due to the spread of new theory and accept need some time, and cloud manufacturing is closely related to the cloud computing has become a hot research topic in the year. After a year, cloud manufacturing research has become diversified, as the research topic, "Cloud Manufacturing" node is the largest, and at the same time with other time points of high frequency keywords have dense continuous connection. In addition to also emerged a large number of hot issues, such as: "System ", "Optimization", Virtualization "," Internet of Thing "," Enterprise ", indicating that this year in the cloud manufacturing research and divergence in the study of. In 2013, the high frequency words appear: "Design", "Genetic Algorithm", "Architecture," Challenge ", "Grid System", Inherit the exploratory research in 2012, and study toward the cloud design and cloud manufacturing architecture. In 2014, the emergence of the "Service", "Service Composition", "Platform", "Ontology", "Resource", "Model", "Internet", "Technology", "Cloud", "Environment" and other words, which means that cloud manufacturing concept, model, key technology and cloud thinking, resource allocation, network environment, and cloud platform, cloud service composition is a research hotspot in the period of time. In 2015, there were a lot of words, such as "Management", "Framework", "Algorithm", "Big Data", "of the Art", "Virtual Enterprise", "Selection", "Network", can be seen that the research focus of this stage is the cloud management, technical architecture, algorithm, data. Process design, virtual enterprise etc. From 2016 -2017, high frequency nodes are very few, only "Simulation", "Thing" these two keywords, indicating that the two years of cloud manufacturing research hotspots are scattered, has not formed a relatively focused research issues. In addition, after 2010, the connection between the time periods is very much, indicating that the inheritance relationship between these research areas is strong, and there is a strong internal logic relationship between the research topics. 


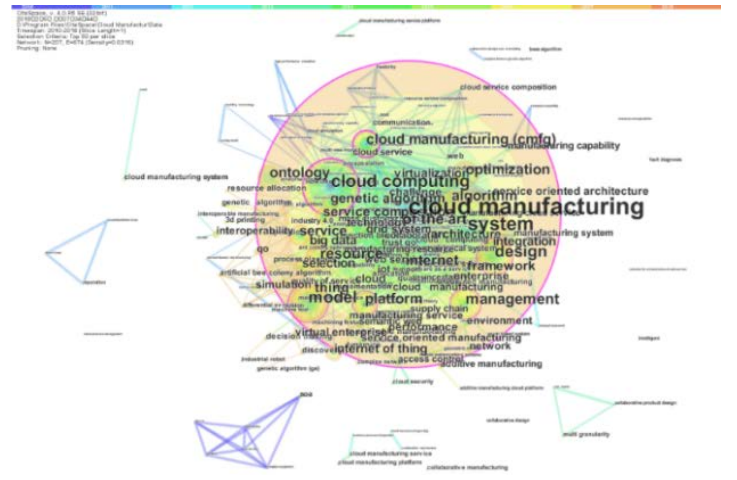

Figure 5. Key words co-occurrence network map

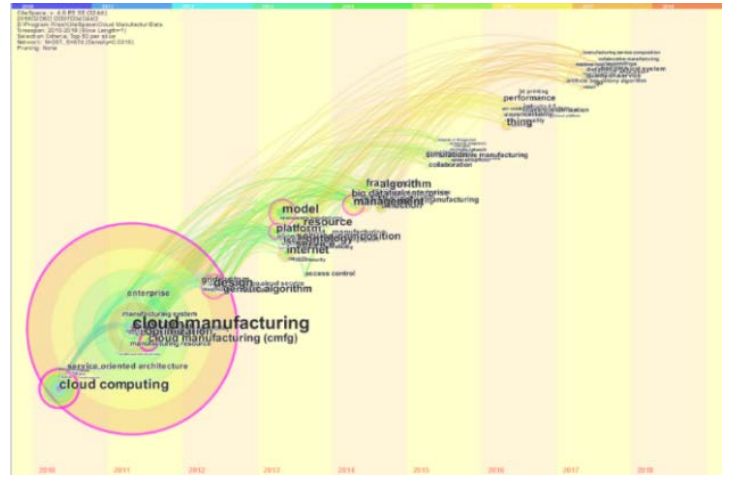

Figure 6. Keywords timing network diagram

\section{Conclusion}

This paper takes the related literature of cloud manufacturing research as the research object and takes the core collection of WOS database as the data source. Through the bibliometrics analysis, this paper shows the trend of the annual growth rate of document volume, the annual input volume of authors, the participants of single papers, the distribution of major research institutes and researchers, combing the knowledge base and innovation path of cloud manufacturing research, and analyzing the hot issues of the research, provide important reference for follow-up researchers to grasp the scientific development and research directions in this field.

From the time distribution point of view, cloud manufacturing research not only the number of scientific research and scientific research groups growing, but also put more scholars and more and more researcher attention. In terms of spatial distribution, more and more research institutes and scholars around the world are concerned about the research of cloud manufacturing, and they are moving toward a high level, deep and comprehensive direction.

From the point of view of knowledge, the documents were cited in the network atlas to show the important researchers in the field of cloud manufacturing research and the year in which they published important scientific and technical documents, all of these are important knowledge bases and sources of knowledge in this field. The centrality of the literature intermediary is another way to identify important scientific and technological literature. From different perspectives, it is systematically searched for important scientific and technological literature in this field.

From the hot point of view, the high frequency keyword table and keyword co-occurrence network map to show the research hotspot of cloud manufacturing together. In the time-series network of key words, cloud manufacturing research hot spots constantly keep pace with the times.

\section{References}

[1] Li, B.H., Zhang, L., Ren, L., et a1. (2011) Computer Integrated Manufacturing Systems, 17, 449.

[2] Li, B.H., Zhang, L., Wang, S.L., et a1. (2010) Computer Integrated Manufacturing Systems, 16, 1.

[3] Li, X.B., Yin, C., Yin, S. (2014) Computer Integrated Manufacturing Systems, 20, 2164.

[4] Xu, X., (2012) Robotics and Computer Integrated Manufacturing Systems, 28, 75.

[5] Li, C.Q., Shang, Y.L., Hu, C.Y. (2011) Combined Machine Tool and Automatic Machining Technology, 7, 104.

[6] Tao, F., Zhang, L., Guo, H., et al. (2011) Computer Integrated Manufacturing Systems, 17, 477.

[7] Ren, L., Zhang, L., Wang, L.H., et al. (2017) International Journal of Computer Integrated Manufacturing, $30,501$.

[8] Wu, D.Z., Greer, J.M., Rosen, D.W., et al. (2013) Journal of Manufacturing Systems, 2013, 564.

[9] Li, B.H., Zhang, L., Ren, L., et al. (2012) Computer Integrated Manufacturing System, 7, 1345.

[10] Zhan, D.C., Zhao, X.B., Wang, S.Q., et al. (2011) Computer Integrated Manufacturing System, 3, 487.

[11] Adamson, G., Wang, L.H., Holm, M., et al. (2017) INTERNATIONAL JOURNAL OF COMPUTER INTEGRATED MANUFACTURING, 30, 347-380.

[12] Qi, E.S., Li, T.B., Liu, L., et al. (2015) Industrial Engineering and Management, 20, 8. 
[13] Yi, S.P., Liu, M., Wen, P.H. (2016) Computer Integrated Manufacturing Systems, 22, 871.

[14] Liu, Z.Y., Chen, Y., Hou, H.Y., Beijing: people's publishing house, 2008, 3.

[15] Zhang, X., Gao, Y., Yan, X., et al. (2015) Computers in Human Behavior, 51, 803.

[16] Lin, Z., Wu, C., Hong, W. (2015) Acta Ecologica Sinica, 35, 142.

[17] Xu, Q., Zhang, W., Hu, L., et al. (2012) Aasri Procedia, 1, 359.

[18] Bornmann, L., Marx, W. (2012) Journal of Informetrics, 6, 285.

[19] Choe, H., Lee, D.H., Kim, H.D., et al.(2016) Renewable \& Sustainable Energy Reviews, 55, 361.

[20] Chen, C.M., (2009) Journal of the American Society for Information Science \& Technology, 57, 359.

[21] Small, H. (1973) Journal of the American Society for Information Science, 24, 265.

[22] Navonil, M., Nik, B., Simon, J.E.T., et al. (2013) Procedia Computer Science, 19, 586.

[23] Xie, W.H., Dong, C., Li, Z.S. (2017) Journal of Information Science, 37, 126.

[24] Hsiao, C.H., Yang C., (2011) International Journal of Information Management, 31, 128.

[25] Song, G.T., Tian, P., Tang X.J., (2015) Modern Information Technology, 35, 164.

[26] Özçınar, H., (2015) Teaching \& Teacher Education, 47, 42.

[27] Ma, Y.C., Huang, K.C. (1999) Information Science, 17, 625.

[28] Zhang, M., Liu, Y.P., Zhu, M.X. (2016) Information Science, 34, 158 